\title{
A computer controlled venepuncture system for blood test
}

Saito/Hirokazu, Miyajima/Kumiko, Kudo/ Hiroyuki and Mitsubayashi/Kohji

Institute of Biomaterials and Bioengineering, Tokyo Medical and Dental University

2-3-10 Kanda Surugadai, Chiyoda-ku, Tokyo 101-0062 Japan

In clinical situation, blood sampling is frequently performed for blood test. Recently, most procedures of blood test have been automated and the test system is developing on a chip-sized structure such as $\mu$-TAS. Achievement of complete automatic blood test system combined with venepuncture device and blood test system would bring decrease of blood sampling volume and realization of home blood test [1]. As an automatic venepuncture device, we developed a control system of needle puncture that measured force and electric impedance acting on a needle tip. To obtain information that the needle tip pierced into the blood vessel, the changes in puncture force and electric impedance were evaluated $[2,3]$.

A medical needle was set on a device for measuring puncture force with a loadcell, and moved by a linear stage as shown as Fig.1. A disk type electrode (Teflon® coated wire with a bared tip) was placed inside the needle. A tape formed $\mathrm{Ag} / \mathrm{AgCl}$ electrode of impedance meter was attached to skin of a subject. The needle was moved with an ultrasonically driven linear stage controlled by a personal computer. When the needle punctured the vein, blood flowed into the needle and caused a short circuit between the skin electrode, tissue, blood and the disk type electrode. The change in electric impedance from the open circuit to the shorted circuit was measured as $\Delta \mathrm{Z}$ via a differentiating circuit in the electric impedance meter. The puncture experiments were carried out on the vein of a rabbit's ear, which was fixed with an elastic bandage. The needle punctured the rabbit's ear vein at a speed of $2.5 \mathrm{~mm} / \mathrm{s}$ and at an angle of about $15^{\circ}$ to the skin surface. The experimental protocol was designed to minimize pain, conforming to the guidelines for animal experimentation at Tokyo Medical and Dental University.

Fig.2 shows a typical example of changes in puncture force and $\Delta Z$ for successful puncture to the rabbit's ear vein. The puncture force increased gradually when the tip of the needle touched the skin. Then a peak in puncture force, caused by piercing the skin and the vessel wall, was observed. The peak value of puncture force was varied considerably, and sometimes could not be observed when the needle pressed the vessel. On the other hand, $\Delta \mathbf{Z}$ decreased suddenly from zero to a large negative value after the force peak when the needle punctured the vein wall. The moment of puncturing blood vessel could be identified by blood flowing into the needle and was not affected by the mechanical condition of puncturing. Although a peak of force appeared, $\Delta \mathbf{Z}$ did not change when the needle punctured beside the vein. It suggests that the combination of puncturing force and $\Delta Z$ would provide information on failure to puncture the vein. As future developments, a breakless needle puncture with a thin needle and a membrane electrode inside/outside on the needle wall should be required for painless blood sucking and securing the sucking volume.

\section{REFERENCES}

1. Zivanovic A, Davies BL, IEEE Trans Inf Tech in Biomed, 4(1), 8-14 (2000)

2. Saito H, Togawa T, Med Biolog Eng Comp, 43, 240-244 (2005).

3. Saito H, Mitsubayashi K, Togawa T, Sensor Actuat A, 125, 446-450 (2006) 


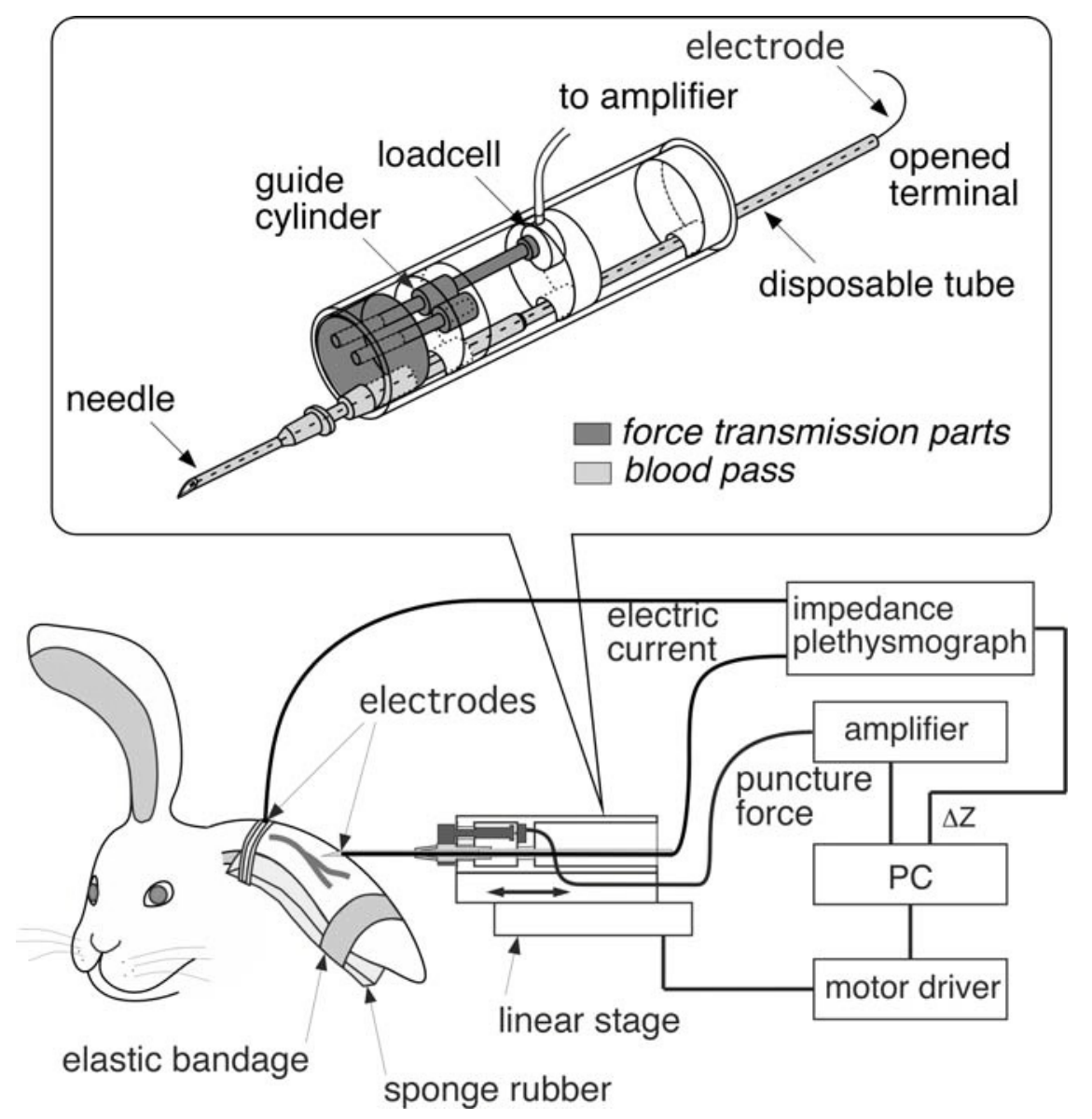

Fig.1 Schematic diagram of an automatic venepuncture system.

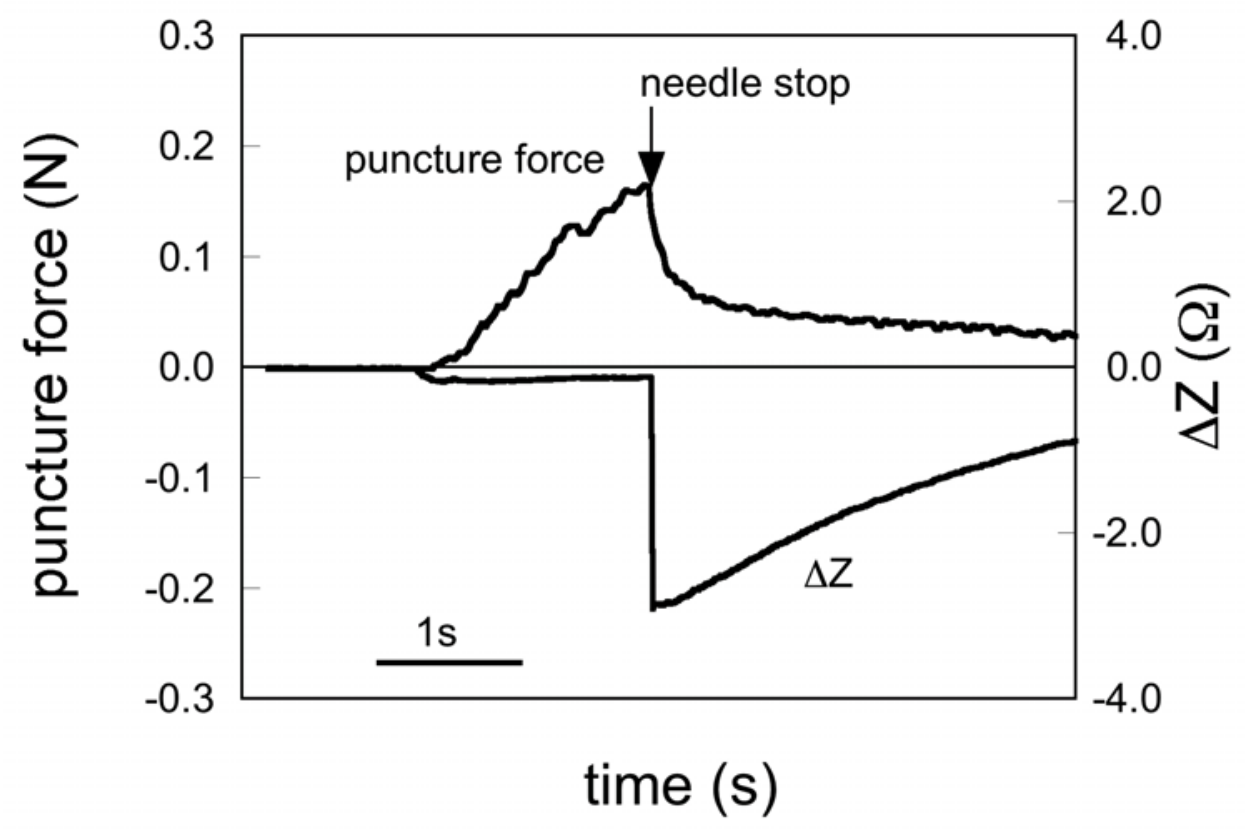

Fig.2 Typical example of successful puncture to the vein. 